\title{
The Development and Standardization of Chinese Urban Traffic Linguistic Landscape
}

\author{
Xiaozhi SU ${ }^{\mathrm{a}, \mathrm{b}, 1}$ and Hong $\mathrm{CHEN}^{\mathrm{a}}$ \\ ${ }^{a}$ College of Transportation Engineering, Chang'an University, Xi'an 710064, Shaanxi, \\ China \\ ${ }^{\mathrm{b}}$ School of Tourism, Xi'an International Studies University, Xi'an 710128, Shaanxi, \\ China
}

\begin{abstract}
Linguistic landscape in a city can not only serve as the informative signs in public area but also record the history and demonstrate the development and modernization with social order and community participation indication. It originates its researching base from social linguistics but develops as interdisciplinary in recent years stimulating deep interests with multi-perspectives. This thesis summarizes the features and developing trends of linguistic landscape researches in and abroad at the first. And with comparative study of typical traffic linguistic landscapes in Chinese and foreign cities, this thesis discusses the framework and categories of traffic linguistic landscape system and its improving role through different stages of time, focusing on the specific features and establishing principles of traffic linguistic landscape in Chinese urban cities.
\end{abstract}

Keywords. Linguistic landscape, traffic linguistic landscape, sustainable development

\section{Introduction}

Lanschaff in German may refer to almost everything on earth, while its counterpart, landscape in English, involves the objects seen on the land. In history, urban cities record the comprehensive and complex development of human cultivation and culture with language as a carrier and medium and linguistic landscapes (LLs), witness and graphers. Of which, Landry and Bourhis [1] whose best quoted definition covered various linguistic tokens, including but not just restricted to government-related inscriptions, street-name signs, commercial signs as well as noticeboards. But it cannot be neglected that Spolsky and Cooper [2] involved the use of multilingual signs in urban cities. Scollon R \& Scollon S W [3] tried systematic researches through intercontinental travel offering the definition of Geosemiotics, which touches the interaction between linguistics and geography. Ben-Rafael E, Shohamy E, Amara M H \& Trumper-Hecht N [4] argued that LLs as symbolic construction of the public space with a case in Israel. Four years later, Jaworski A \& Thurlow C [5] introduced semiotic landscapes through language, image and space. With the contribution of Gorter [6],

\footnotetext{
${ }^{1}$ Xiaozhi Su, College of Transportation Engineering, Chang'an University, Xi'an 710064, Shaanxi, China; E-mail: 335213913@qq.com.
} 
LLs were treated as a new approach for multilingual researches. No doubt that LLs have proven to be intriguing and interesting foci of sociolinguistic research and given language in public spaces indicators broader sociolinguistic processes, tussles, and policies. There have been increasing contributions on the multimode research of LLs worldwide, including China. From the starting research about geographical names by Jianmin Chen in 1979 [7] to geographical distribution of local dialect in Guangdong province by Shangji Situ in1992 [8], Chinese researchers contributed both theoretical and case studies about LLs with a practice on introduction and analysis of foreign researches at the beginning like Tiun, Hak-khiam [9], Siying Cai [10], Guiwen Shang, et al [11], Lisheng $\mathrm{Li}$ [12] and Ming Xu, et al [13] to new perspectives by Junli Ge [14] and Guowen Shang, et al [15] and so on in recent years. That is to say, with the urban development and modernization in China, researchers found multimode in LLs discussion. But this thesis tries for the first time by taking Traffic Language System (TLS), the tool by which traffic managers communicate with travellers, in turn forming Traffic Linguistic Landscapes (TLLs) as a subset of urban LLs to assimilate the regularity of traffic management information and traveller related information with that of language system. In the study the author explains that with the aid of TLS, traffic management information and travellers' information would be translated into traffic information to the users and vice versa. The combination of texts, sounds, signals, pictures and colors with local cultural features in TLS follows uniqueness and specialty from time to time in urban cities. And then with study of typical TLLs in and abroad, this thesis discusses the framework features and establishing principles of TLLs in Chinese urban cities from the perspective of sustainability.

\section{The Definition of TLLs}

Language, from the dictionary, is defined as the spoken or written communication used by a particular country, people and community, etc. And it typically consists of words within a certain regular grammatical and syntactic structure. While in social linguistics, the concept "language" also refers to cognitive faculty that enables humans to learn and use complex communication systems. LLs, with the most quoted definition, cover diverse linguistic tokens, including but not just restricted to government-related inscriptions, street-name signs, commercial signs as well as noticeboards. With technological development, LLs in modern cities also involve new dynamic application like electronic signs, multimedia facilities, etc. And as a subset of LLs, TLLs in general refer to LLs in transportation. It is a collection of symbol system and rule system based on texts, signs colors as well as sounds and videos. On basis of linguistic terms, the application of traffic language can be reflected in the traffic signs, lines and marks as well as other traffic management facilities containing series of traffic and travel related information, which broadly includes road signs and signals, traffic lights, marking lines, hub transfer information and pedestrian guidance, etc. offering information and guidance or management in different travel modes.

\section{Framework and Categories of TLLs}

TLLs form a sub-set of LLs and follow the same systematic rules. According to the morphology guidelines, a linguistics branch concerned with the internal structure of 
words, a morpheme is the smallest meaningful linguistic unit in the grammar of a language, and the same for traffic language, which is special but in common practice of daily travel.

\subsection{Framework of TLLs}

The morpheme in traffic language lies in the basic words, signs, markings or colors that form the "texts" providing carriers of demanded information in the whole travel process. Such morphemes may offer basic management information in transportation but may not interpret a complete and complex "traffic message". Instead, the combination of multiple morphemes can express complete systemic management information in traffic system.

In reality, the combination of morphemes is common to see, which in turn form the "words" (morphemes combination of TLLs), or the bigger grammar units on basis of syntagmatic and paradigmatic principles like what is in the natural language.

\subsection{Categories of TLLS}

TLLs can be categorized according to the using area [16]. If LLs are described as the general set, the TLLs will be at the sub-level.

\subsubsection{TLLs in Motorized Traffic}

TLLs in the motorized traffic system embody marking signs, variable informative boards, stopping guidance and so on. These facilities are composed of the most basic language elements. Motorized traffic generally involves long distance and rapid travel. Based on the characteristics of this kind of travel, there are three types of travel modes: the frequent and fixed route travel, frequent but non-fixed route travel and occasional (time and line) travel. Specially designated TLLs therefore provide information service and establish communication between traffic managers and travellers with regards of different using demands.

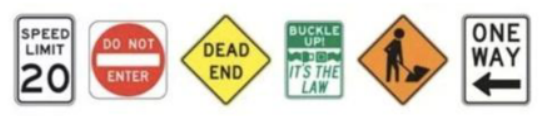

Figure 1. Series of TLLs in motorized traffic. (From Wechat fanyijiang666)

TLLs in figure 1 interpret messages of speed limit, forbiddance of entry, no way out on the road, requirement of safe belt use, way in construction and direction of the only way respectively, which are mainly used with color indication in function in motorized traffic system.

\subsubsection{TLLs in Slow Traffic}

TLLs, with a comparatively small design space in slow traffic system, mainly involve signs and guidance information to bicycle riders and pedestrians. With the function of keeping continuous travel space and reasonable isolation conversion between mass public traffic and motorized traffic, TLLs offer ensure of travel safety and smoothness. 


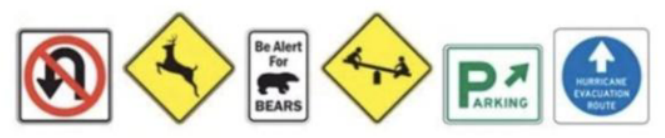

Figure 2. Series of TLLs in motorized traffic and slow traffic. (From Wechat fanyijiang666)

TLLs in figure 2 interpret messages of on turning, alert for animals, concern for people in parks, parking area and direction of evacuation respectively, which indicate the synergistic information in use of both motorized and slow traffic systems.

\subsubsection{TLLs in Mass Transit System}

TLLs in mass transit system which mainly refer to Normal Bus Transit (NBT), Bus Rapid Transit (BRT) and Rapid Rail Transit (RRT) as shown in figures 3-4, can provide travel information and transfer guidance related to public transport.

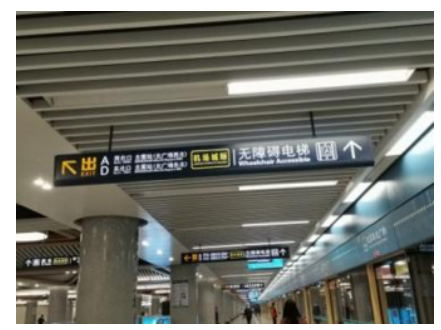

Figure 3. TLLs in L4, Xi'an Metro.



Figure 4. Dynamic TLLs in L4, Xi'an Metro.

\section{Principles for Development and Standardization of TLLs}

LLs are linguistic tokens used in different space including both urban and rural areas in general. With which the use of certain languages can be displayed and interpreted but LLs can not only serve as the informative signs in public area but also record the history and demonstrate the development and modernization in certain areas especially with social order and community participation indication in urban cities.

\subsection{General Features and Establishing Principles of TLLs}

Traffic language is a medium set up by traffic managers to ensure the smooth transfer of information between traffic participants and traffic systems.

The uniformity of traffic language is first embodied in the legality, rationality, compliance and unity of language text in traffic system. At the same time, the uniqueness of the same system and the coordination between different systems are reflected. With the development of economy and technology, the complexity of traffic system is increasing day by day. The setting technology and related standards of traffic language are also put forward.

Secondly, intuitiveness of traffic language includes independence, visibility and clarity. TLLs in urban cities especially need to provide clear, direct and easily accessible traffic information in the physical movement of vehicles and travelers. 
Thirdly, the continuity of TLLs includes physical continuity and normative continuity. It is also the guarantee of the traffic information transmission and traffic service as well as traffic management. In general, the continuity of norms is embodied in the normative continuity of traffic regulations, traffic organization and management measures in TLLs as well as in the standard continuity of setting and application of TLLs.

Fourthly, systematization of TLLs mainly refers to the relationship and compatibility between various factors in traffic language system. Traffic facilities are used by the movement of travelers or certain objects, which is also the use of traffic behavior and process. TLLs in traffic facilities constitute the traffic sign system after systematic integration. And in turn traffic sign system is the foundation of traffic language system, which is a subset of the whole symbol system [17].

\subsection{Specific Features and Standardizing Principles of TLLs in Urban Cities}

One of fundamental features of urban cities is that the city is spatial. Multimodal LLs in urban space produced a semiotic symbolic system which predicts the city through all kinds of language use and design with indication of social order, cultural exchange and urbanization itself. Principles for TLLs establishment and standardization can be put into consideration from geographical, temporal and communicative perspectives.

\subsubsection{Features and Principles from Geographical Perspective}

Language is the most basic tool and convenient medium for information transmission and communication in human society. Languages are created and used in a certain geographical space and naturally associated with "locality" of the connected environment. Traffic language has the commonness and consistency in serving the traffic system, but should clearly show geographical features with the influence of local economy, politics, history and nationality as well as the development of science and technology.

Take crossing marks as an example, they are in practice set specifically considering the elements of color or design patterns in different countries and regions. For example, in Mexico and Brazil, normal zebra crossings are taken place by combination of different shaped patterns, two columns of white direction arrows with horizontal lines for the former and reverse dagger-shaped designs for the latter [18]. In China, the most commonly used crossing mark is white zebra lines. However, with the improvement of the requirement of service consideration and refinement in traffic system, both combination of patterns and colors have been chosen for a change to meet the specific needs in local transportation.

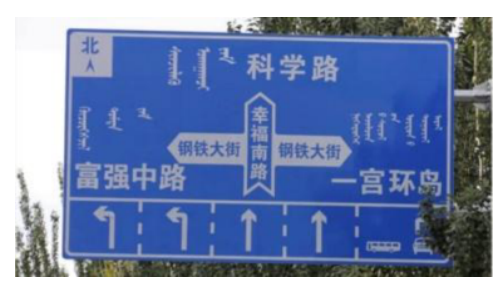

Figure 5. Bilingual Traffic Signs in Baotou City, Inner Mongolia Autonomous Region, China. 


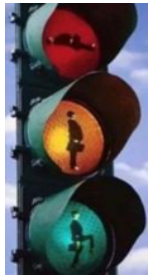

(a)

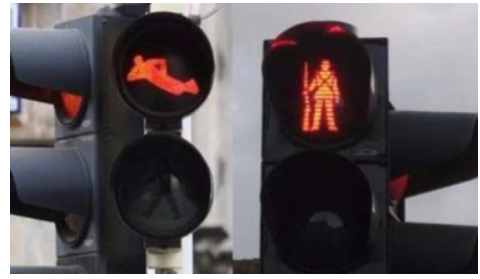

(b)



(c)

Figure 6. Traffic Lights with Local Features in European ITCs (a-c).

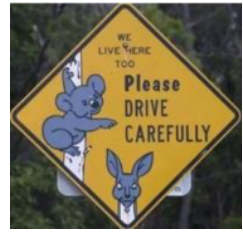

(a)

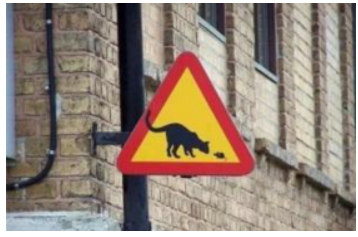

(b)

Figure 7. Representative Street Signs in ITCs (a-b).

Bilingual traffic signs in figure 5 shows specific features of local language use and situation in Chinese cities especially those with ethnical groups' habitats. And with the development of unban cities and tourism, there is the great increasing demand of precise and meticulous service in all kinds of perspectives including traffic system. Bilingual or multilingual traffic signs are in urgent need. Traffic lights with local features of European cities in figures 6(a)-(c) add specific strangeness and new possibility of attraction to both international and local travelers. Representative street signs in figures 7(a)-(b) with typical or even unique local images can offer quick and direct reminding of the city.

Characteristic modes of geographical features of TLLs refer to their special distribution and multimode combination of diverse traffic signs need to be set with matching connection with other facility systems, which establish TLLs with strong regional cultural characteristics in different traffic applications to highlight distinct geographical features in different cultural dimensions and interfaces.

\subsubsection{Features and Principles from Temporal Perspective}

The traffic language used in different geographical spaces is not fixed or unchanging. With the development of the times, the progress of science and technology and the increase of the complexity of urban transportation, the specific content, practical use and settings of traffic language have undergone corresponding changes, showing different times. First of all, the text content of traffic language will transmit information changed accordingly. The semantics of the texts may be the same, but the change of wording shows clearer harmony between the mass transit system and the motorized traffic system, with more consideration and politeness in words choice.

Temporal features of TLLs in urban cities also reflect in the provision of accurate and refined traffic information and service. In response to the more complex traffic demands, new types of traffic signs appear constantly. 
Finally, with the development of new-tech and sustainable demands, the setting of TLLs presents timely and intelligent characteristics. The use of new environmentfriendly materials, intelligent programs and big data make traffic language timely and accurate transmission of traffic information.

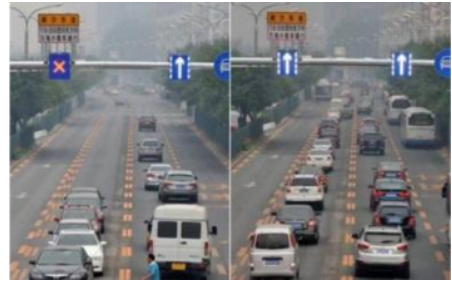

Figure 8(a). Reversible lanes in Chinese ITCs.

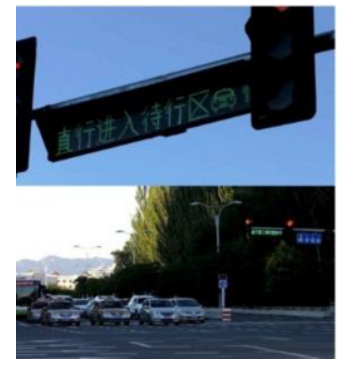

Figure 8(b). Led-traffic signs in Chinese ITCs.

Reversible lanes or tidal flows in Chinese urban cities in figure 8(a) are set to relieve road congestion especially to meet traffic demand in morning-evening rush hour. Led-traffic signs in figure 8 (b) offer dynamic traffic information and service.

\subsubsection{Features and Principles from Communicative Perspective}

The formation of language and the development of culture interact and restrict each other. Language is a special cultural phenomenon, and culture relies on language as a carrier to spread. In the same way, traffic language is also an important part of traffic culture, and more embodied in the category of artifacts culture. The process of text setting and information transmission of traffic language is also the inheritance and transmission of its specific geographical culture according to different time. In urban cities, the number of globally cross-cultural communication is increasing geometrically. The service and management of the integrated traffic system, which is constantly improved and gradually developed is the foundation to promote this kind of communication.

The communicative function of traffic language is conductive for the exchanges between people in different countries or regions.

\section{Conclusions}

Language is a kind of multi-layered symbolic device. Traffic language forms a subsystem of signs and marks which serve at every level of the complex traffic system. In urban TLLs system, the combination of sounds, symbols, marks and colors as well as geographical and cultural features does have irreplaceable uniqueness. Such nonmaterialized elements carry inheritance massages through certain space and time with specialized law and principles and have epochal characteristics at different stages. As the important content of traffic management and service system, traffic language system and other urban landscape construction should be integrated and standardized and the TLS of urban cities should carry multi-dimensional and intelligent characteristics to meet the need of urban development. That is to say, in addition to the 
physical functions and visual effect, TLLs with multi-lingual standardization and newtech assistance can catch up with the internalization, communication and modernization of Chinese urban cities. With the development of internet, the setting and standardization of language landscape in traffic field should follow the principles of sustainable development to promote the construction of traffic language landscape systematically and sustainably.

\section{Acknowledgement}

We wish to acknowledge the following funding: Scientific Research Program funded by Shaanxi Provincial Office for Philosophy and Social Science (Program No. 2015H010) and Xi'an International Studies University (Program No. 18XWD16).

\section{References}

[1] Landry R and Bourhis RY. Linguistic landscape and ethnolinguistic vitality: An empirical study. Journal of Language and Social Psychology. 1997 Mar; 16(1): 23-49.

[2] Spolsky B \& Cooper R. The language of Jerusalem. Oxford University Press; 1991.

[3] Scollon R \& Scollon SW. Discourses in place: Language in the material world. Routledge; 2003.

[4] Ben-Rafael E, Shohamy E, Amara MH \& Trumper-Hecht N. Linguistic landscape as symbolic construction of the public space: The case of Israel. International Journal of Multilingualism. 2006 Apr; 3(1): 7-30.

[5] Jaworski A \& Thurlow C. Semiotic landscapes: Language, image, space. Continuum International Publishing Group; 2010; p 1-40.

[6] Gorter D. Linguistic landscapes in a multilingual world. Annual Review of Applied Linguistic. 2013 Nov; 33: 190-212.

[7] Chen JM. Discussion on geographical names. Social Science Front. 1979 Aug; (4): 336-337.

[8] Situ ShJ. The geographical distribution of cantonese dialect and its cultural landscape. Southeast Culture. 1992 Aug; (Z1): 174-186.

[9] Sun L. Linguistic landscape and language maintenance planning. Journal of National Taitung Teachers College. 2009 Dec; 42(6): 153-156.

[10] Cai SY. The preliminary exploration of geosemiotics-Text analysis in space. Journal of Educational Institute of Jilin Provence. 2012 Aug; (8): 125-127.

[11] Shang GW, Zhao ShH. Linguistic landscape studies: Perspective, theories, and approaches. Foreign Language Teaching and Research. 2014 Mar; (2): 214-223.

[12] Li LSh. A review of international linguistic landscape studies and its implications. Journal of Beijing International Studies University. 2015 Apr; (4): 1-7.

[13] $\mathrm{Xu} \mathrm{M}$ and $\mathrm{Lu} \mathrm{S}$. Research progress and prospect of urban linguistic landscape. Human Geography. 2015 Feb; (4): 1-7.

[14] Ge JL. Language and space: A perspective of linguistic landscape. Journal of Beijing International Studies University. 2016 Oct; 38(4): 68-80.

[15] Shang GW and Zhou XW. Peripheral linguistic landscape: Sign types, features and research perspectives. Chinese Journal of Language Policy and Planning. 2020 Jun; 5(4): 37-47.

[16] Zu YCh, Liu DB, Wang YX, Li Y. Classification and usage of the standards of road traffic signs and marketings. China Public Security (Academy Edition). 2014 Mar; (1): 71-74.

[17] Fedinand DS. Course in general linguistics. The Commercial Press; 2001.

[18] Duan LR, Mao LZ. Analysis on the characteristics and setting principles of urban road traffic language. Management of Transportation. 2013 Jun; (6): 83-89. 